ERCP alone for stone disease. This likely reflects the high complexity of stone cases necessitating cholangioscopy. Of note, the radiation exposure/minute for ERCP with cholangioscopy in stone disease is no different to conventional ERCP. Our data on cholangioscopy in stricture assessment suggests that this may enhance diagnostic information (ie direct visualisation) without adding procedure time or radiation exposure.

\section{PTH-013 A VALIDATED COMFORT SCORE FOR GASTROSCOPY TOLERATION}

F Betteridge*, E Saunsbury, L Materacki, K Yong, D Murugiah, B Colleypriest. Royal United Hospital, Bath, UK

10.1136/gutjnl-2018-BSGAbstracts.35

Introduction Whilst comfort scoring is an auditable standard for quality in colonoscopy, there is currently no widely accepted comfort score for gastroscopy. We developed a five point gastroscopy toleration score (GTS) and validated correlation of scoring between endoscopists, patients and nursing staff.

Methods A GTS was developed via consensus on a gastroscopy list (figure 1). Trial 1 of the finalised 'Bath GTS' involved a double blind collection from the endoscopist and two nurses during 47 gastroscopies to determine inter-observer correlation between staff. Trial 2 involved a double blind collection from the endoscopist, nurse and unsedated patient during 50 additional cases. Krippendorff's alpha $(\alpha)$ coefficient was calculated to assess inter-rater reliability. We then analysed sub-group matches between endoscopist-nurse, endoscopistpatient and nurse-patient.

Results A total of 97 gastroscopies were included in the analysis. In Trial 1 the inter-rater correlation between nurses and endoscopist was excellent (Krippendorff's $\alpha=0.811$ (95\% CI $0.73-0.88)$ ). There were no matched scores in 1 case $(2.1 \%)$, at least two matched scores in 46 cases $(97.9 \%)$ and three matched scores in 34 cases (72.3\%).

\section{Bath Gastroscopy Toleration Score (GTS)}

0- Still throughout, may retch at intubation

1- Minimal retching $(<25 \%$ of procedure time)

2- Frequent retching ( $>25 \%$ of procedure time)

3- Attempts to handle and/or remove gastroscope

4- Extubates themselves or withdraws consent

\section{Abstract PTH-013 Figure 1}

Trial 2 demonstrated at least two matched scores in all 50 cases $(100 \%)$ and three matched scores in 39 cases $(78 \%)$ with excellent correlation between raters $(\alpha=0.833$ (95\% CI $0.75-0.90)$ ). Subgroup analysis demonstrated that of the gastroscopies with only two matched scores, endoscopist-nurse matches totalled 8 cases $(72.3 \%)$, endoscopist-patient matches totalled 2 cases (18.2\%) and nurse-patient matches 1 case
(9.10\%). Krippendorff's $\alpha$ for these subgroups were 0.930 (95\% CI $0.84-1.00), 0.800 \quad(95 \%$ CI $0.63-0.93)$ and 0.774 (95\%CI 0.60-0.92) respectively. Final analysis comparing endoscopist-nurse matched scores from all 97 gastroscopies showed significant agreement ( $\alpha=0.858$ (95\%CI $0.81-0.91)$ ).

Conclusions We have presented a simple yet effective GTS which was validated for inter-observer correlation between endoscopists and nurses with statistically significant agreement. Furthermore, there is excellent correlation when the GTS from unsedated patients is compared with that of staff $(\alpha=0.833$ (CI $0.75-0.90)$. Given the agreement between unsedated patients and staff regarding procedure toleration, this scoring system could be applied to sedated patients. We would suggest that the Bath GTS is adopted as a validated national auditable outcome for gastroscopy with further work in progress to determine the standard.

\section{PTH-014 POST-POLYPECTOMY SURVEILLANCE IN THE ENGLISH BOWEL CANCER SCREENING PROGRAMME: A PROSPECTIVE COHORT STUDY, PRELIMINARY RESULTS}

${ }^{1,2}$ Stewart Bonnington*, ${ }^{2}$ Linda Sharp, ${ }^{1,2}$ Matt Rutter. ${ }^{1}$ North Tees and Hartlepool NHS Trust, Stockton-on-Tees, UK; ${ }^{2}$ Newcastle University, Newcastle Upon Tyne, UK

\subsection{6/gutjnl-2018-BSGAbstracts.36}

Introduction The English Bowel Cancer Screening Programme (BCSP) offers individuals aged 60 to 74 years guaiac FOB testing $(\mathrm{gFOBt})$, with an invitation for colonoscopy if positive. Of more than 300000 individuals who have attended for colonoscopy, over 45000 attended post-polypectomy surveillance (PPS) after having intermediate (IR) or high risk (HR) adenomas detected and resected at screening. It is established that screening reduces mortality from colorectal cancer (CRC). However, robust evidence to support PPS is lacking.

Methods Details were extracted from the BSCP database for individuals who attended PPS from the start of the BCSP in 2006 until 3/1/2017. Data were analysed using Stata 14.

Results 67,435 PPS episodes were performed in 45151 individuals. $60 \%$ of individuals had attended only 1 PPS episode, $34 \%$ attended $2,5 \%$ attended 3 , and $0.4 \%$ attended 4 or 5 .

PPS episodes per year greatly increased over the study period. In 2008, 453 episodes were attended, rising to 13698 in 2016 (figure 1).

$70 \%$ of those attending PPS were male. $44 \%$ were aged 60 64 at the time of index screening, 43\% aged 65-69, and 10\% aged 70-74. The oldest age group had a higher proportion $(68 \%)$ of HR individuals than in the younger groups.

Screening risk category varied with gender. Overall, $40 \%$ of females and $51 \%$ of males were HR (figure 2).

Overall, the findings at first PPS demonstrate a high proportion of individuals with no further adenoma found (table 1).

Conclusions PPS accounts for an increasing proportion of endoscopy workload in the BCSP and more broadly in the UK and internationally. These results demonstrate a low proportion of CRC or IR or HR adenomas diagnosed at PPS in the BCSP.

Individuals with HR adenomas at screening more often had further adenomas detected at first PPS when compared to those with IR adenomas at screening. HR individuals also had a higher probability of IR or HR adenomas at first PPS. CRC diagnosis at first PPS was low in both groups $(\leq 0.5 \%)$.

Further evaluation of the BCSP database is ongoing in order to identify subgroups most likely to benefit from PPS. 